\title{
Spectroscopie d'émission du noyau Raphe Dorsalis chez le rat non anesthésié sous excitation laser UV 300-355 nm
}

\author{
S. Mottin, P. Laporte, M. Jouvet* et R. Cespuglio* \\ Laboratoire TSI, CNRS UMR 5516, 42023 Saint-Etienne cedex 02, France \\ * Laboratoire d'Onirologie Moléculaire, INSERM U52, 69373 Lyon cedex 08, France
}

\begin{abstract}
Résumé : La spectroscopie d'émission de fluorescence du noyau Raphe Dorsalis a été étudiée à différentes longueurs d'onde d'excitation dans la gamme $300-330 \mathrm{~nm}$ et aussi à $355 \mathrm{~nm}$ chez le rat libre de tous mouvements et éveillé. Les résultats expérimentaux montrent des changements importants du spectre d'émission .
\end{abstract}

La spectroscopie d'émission du tissu cérébral reste très largement mal connue. Les études précédentes ont surtout été réalisées avec deux types de sources : la lampe mercure $(366 \mathrm{~nm})$ [1] et le laser azote $(337 \mathrm{~nm})[2,3]$. Ces études antérieures ont convergé vers une attribution de l'émission observée vers $480 \mathrm{~nm}$ comme étant majoritairement due au fluorophore endogène NADH. Nous avons voulu entreprendre l'investigation des propriétés de fluorescence du tissu cérébral chez le rongeur éveillé à des longueurs d'onde plus basses afin d'exciter d'éventuels fluorophores différents. La sensibilité à la longueur d'onde d'excitation est, pour la première fois à notre connaissance, ici clairement mis en évidence.

Nous présentons les spectres d'émission de fluorescence du tissu cérébral consécutifs à une excitation laser UV accordable $300-330 \mathrm{~nm}$ et complétée par une excitation à $355 \mathrm{~nm}$.

La cible cérébrale choisie est le noyau Raphe Dorsalis (nRD) où plus de $40 \%$ des neurones sérotoninergiques sont présents. Cette structure de moins de $0.2 \mathrm{~mm}^{3}$ est clairement impliquée dans les mécanismes qui sous tendent le cycle veille-sommeil. Notre étude s'insère dans le cadre de la biophotonique cérébrale et plus particulièrement dans le développement de nouveaux outils de diagnostic in vivo et in situ dans le domaine des neurosciences.

Le dispositif expérimental utilisé consiste en :

- une source laser accordable dans l'UV (OPO901; BMI, France) constitué d'un ensemble YAG triplé + OPO + doubleur, de cadence $30 \mathrm{~Hz}$, nanoseconde (5-6ns) et utilisée à une puissance moyenne réduite $\approx 1 \mathrm{~mW}(\approx 33 \mu \mathrm{J} / \mathrm{tir})$;

- un ensemble optique de couplage comprenant notamment Laser/fibre/animal/monochromateur est utilisé pour l'expérience et a été décrit précédemment [3]

- une détection par caméra à balayage de fente (StreakScope, Hamamatsu, Japon) couplée à un monochromateur (SPEX 270, Jobin-Yvon, France), et utilisée en mode comptage de photoélectron sur une gamme de 10ns pleine échelle.

L'ensemble laser permet de générer d'une part du 355nm (YAG triplé) et d'autre part dans de bonnes conditions la gamme 295-330 nm après doublement du signal issu de l'OPO.

Les spectres d'émission du nRD (Figure 1) mesurés in vivo. à différentes longueurs d'onde d'excitation ont été mesurés dans cette gamme d'excitation. Le temps de comptage pour chaque spectre est de 1 minute. Les résultats sont lissés par 20 points contigus avec le logiciel Kaleidagraph 3.0.1 édité par Abelbek Software, USA.

Le bruit mesuré en plongeant la fibre optique dans de l'eau milliQ (Millipore) reste très faible quelle que soit la longueur d'onde d'excitation. La fluorescence d'une solution in vitro de NADH à $80 \mu \mathrm{M}$ mesurée dans les mêmes conditions, a été reportée sur la figure 1 . 
Le passage du pic apparent ' $380 \mathrm{~nm}$ ' ( voir courbes avec $\lambda_{\text {exc }}=300-304 \mathrm{~nm}$ ) au pic ' $480 \mathrm{~nm}$ ' $\left(\lambda_{\text {exc }}=324 \mathrm{~nm}-355 \mathrm{~nm}\right)$ est clairement marqué.

Les changements spectraux sont importants sur le domaine d'excitation $300-355 \mathrm{~nm}$. Ces spectres d'émission sont non corrigés des effets chromatiques des différents composants utilisés. Le composant le plus critique est le miroir dichroïque; celui ci étant $R$ max pour l'UV et $R=50$ $\%$ à $360 \mathrm{~nm}$. La partie du spectre d'émission inférieure à $370 \mathrm{~nm}$ est donc diminuée en intensité. Malgré tout l'information sur la sensibilité de la longueur d'onde d'excitation est bien mise en évidence. Le paramètre qu'est la longueur d'onde d'excitation, se révèle donc être très sensible pour les tissus cérébraux. Il apparait à travers cette étude préliminaire que le point de basculement se situe vers $327 \mathrm{~nm}$. Il n'est pas aisé d'interpréter cette sensibilité aux longueurs d'onde d'excitation dans cette gamme 300-355nm à la seule vue des spectres d'émission. La variation de l'absorption du NADH in vitro dans cette gamme étant d'un facteur 2.5, elle n'est pas suffisante pour expliquer cette extrème sensibilité. La multitude des effets optiques d'intéraction laser/tissu reste pour une large part à éclaicir [2, 4]:

(1)absorption directe et réabsorption de l'émission dues aux chromophores endogènes des différents compartiments cellulaire, extracellulaire et sytème circulatoire, en particulier la bande de Soret des hémoglobines

(2)fluorescence et déclins non radiatifs (transfert d'énergie, inhibition de fluorescence...)

(3)réflexion surfacique

(4) diffusion de la lumière.

Cette complexité de la biophotonique est un élément qui nécessite des approches complémentaires. Une des approches sur lesquelles nous nous orientons est la résolution temporelle picoseconde qui nécessite un laser femtoseconde/picoseconde accordable dans l'UV. Grâce à cette approche spectro-temporelle nous pensons pouvoir mesurer plus précisément les variations de la composition biochimique des différents compartiments dans différentes situations (vigilance, sommeil, sommeil paradoxal...) en fonction des besoins des neurophysiologues.

Remerciements: DRET (contrat 93057), BMIndustrie, Hamamatsu, région Rhone-Alpes, CNRS.

\section{Références :}

[1] Chance B., Cohen P., Jobsis F., Schoener B., Science 137, (1962) 499-508

[2] Mayevsky A., Brain Res. Rev. 7, (1984) 49-68.

[3] Mottin S., Laporte P., Cespuglio R., Jouvet M., Annales de Physique 19, (1994) C1 187 194

[4] Durkin A.J., Jaikumar S., Richards-Kortum R., Appl. Spectrosc. 47, (1993) 2114-2121

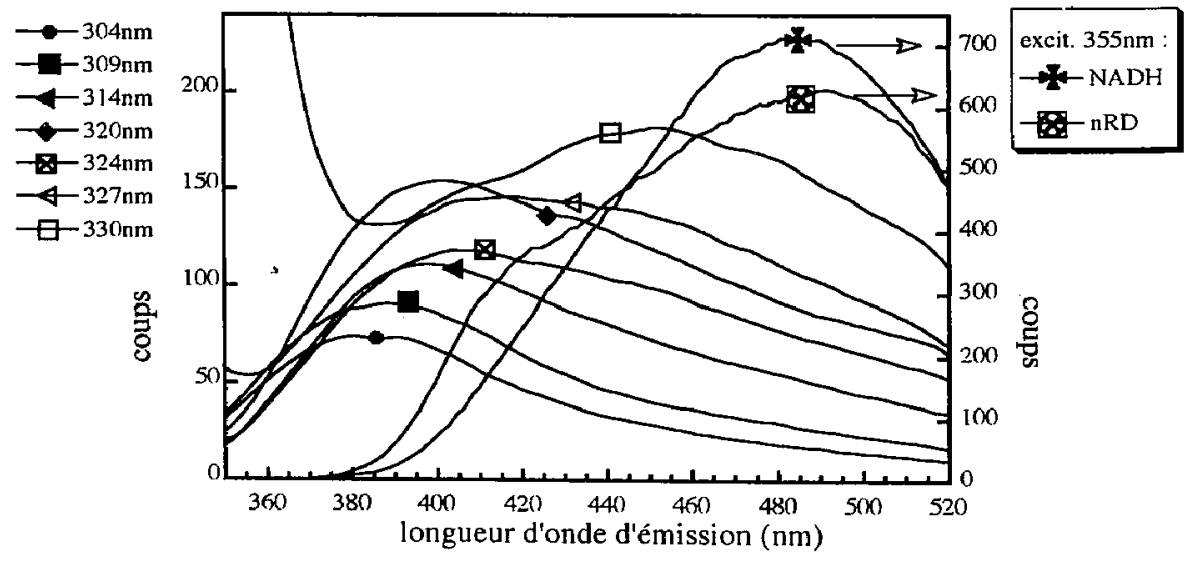

Figure 1: Spectres d'émission du noyau Raphe Dorsalis chez le rat vigile et du $\mathrm{NADH}$ en solution pour différentes longueurs d'onde d'excitation. 